\title{
Corrigendum to "miR-183-5p Is a Potential Molecular Marker of Systemic Lupus Erythematosus"
}

\author{
Shaolan Zhou $\mathbb{D}^{1,2}$ Jing Zhang, ${ }^{1,3,4}$ Pengfei Luan $\mathbb{D}^{1,3}$ Zhanbing Ma ${ }^{\mathbb{D}},{ }^{1,3}$ Jie Dang $\mathbb{D}^{1,3}$

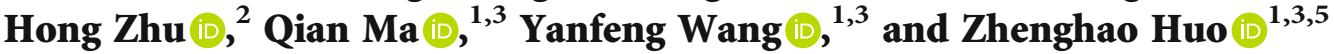 \\ ${ }^{1}$ Department of Medical Genetics and Cell Biology, College of Basic Medicine, Ningxia Medical University, Yinchuan, \\ Ningxia, China \\ ${ }^{2}$ Department of Rheumatology, General Hospital of Ningxia Medical University, Yinchuan, Ningxia, China \\ ${ }^{3}$ Key Laboratory of Fertility Preservation and Maintenance (Ningxia Medical University), Ministry of Education, Yinchuan, \\ Ningxia, China \\ ${ }^{4}$ Ningxia Key Laboratory of Cerebrocranial Diseases, Ningxia Medical University, Yinchuan, Ningxia, China \\ ${ }^{5}$ Department of Biology, Gansu Medical College, Pingliang, Gansu, China \\ Correspondence should be addressed to Zhenghao Huo; huozhh@163.com
}

Received 14 August 2021; Accepted 14 August 2021; Published 11 September 2021

Copyright ( 2021 Shaolan Zhou et al. This is an open access article distributed under the Creative Commons Attribution License, which permits unrestricted use, distribution, and reproduction in any medium, provided the original work is properly cited.

In the article titled "miR-183-5p Is a Potential Molecular Marker of Systemic Lupus Erythematosus" [1], Figure 8 was formatted incorrectly. The authors have corrected this error and provided the correct figure as follows: 


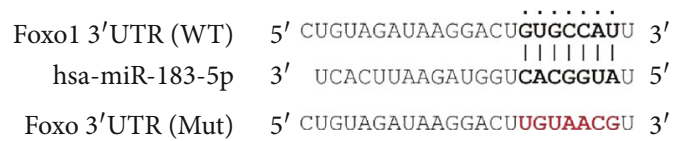

(a)

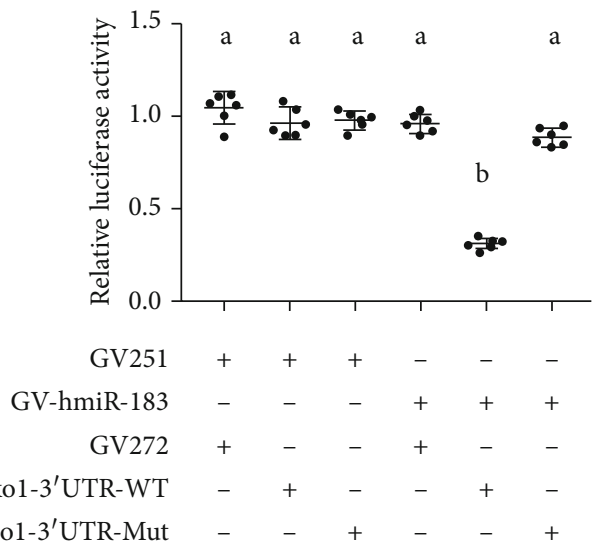

(b)

FIgURE 8: miR-183-5p directly targets Foxo1 3'UTR. (a) The binding site of miR-183-5p and the position 226-242 of Foxo1 3'UTR wild type (WT) and mutant type (mut). (b) miR-183-5p ectopic expression significantly inhibited luciferase activity of the wild-type Foxo1 $3^{\prime}$ UTR reporter plasmid in comparison with the mutated counterpart. Groups labelled with different letters are statistically different from each other $\left({ }^{*} p<0.05\right)$. Differences between groups were analyzed for statistical significance by ANOVA with Fischer's probable leastsquare difference post hoc test.

\section{References}

[1] S. Zhou, J. Zhang, P. Luan et al., "miR-183-5p Is a Potential Molecular Marker of Systemic Lupus Erythematosus," Journal of Immunology Research, vol. 2021, 11 pages, 2021. 